\title{
Efficient Solution of Optimal Multimarket Electricity Bid Models
}

\author{
Cristina Corchero ${ }^{\# 1}$, F.-Javier Heredia ${ }^{\# 2}$, Eugenio Mijangos ${ }^{* 3}$ \\ \# Group on Numerical Optimization and Modeling (gnom.upc.edu) \\ Dept. of Statistics and Operational Research, Universitat Politècnica de Catalunya \\ C5 building, Campus Nord, Jordi Girona 1-3, 08034 Barcelona, Spain. \\ ${ }^{1}$ cristina.corchero@upc.edu \\ ${ }^{2}$ f.javier.heredia@upc.edu \\ ${ }^{*}$ Dept. of Applied Mathematics and Statistics and Operations Research, University of the Basque Country \\ P.O. Box 644 E-48080 Bilbao, Spain. \\ 3 eugenio.mijangos@ehu.es
}

\begin{abstract}
Short-term electricity market is made up of a sequence of markets, that is, it is a multimarket enviroment. In the case of the Iberian Energy Market the sequence of major short-term electricity markets are the day-ahead market, the ancillary service market or secondary reserve market (henceforth reserve market), and a set of six intraday markets. Generation Companies (GenCos) that participate in the electricity market could increase their benefits by jointly optimizing their participation in this sequence of electricity markets. This work proposes a stochastic programming model that gives the GenCo the optimal bidding strategy for the day-ahead market (DAM), which considers the benefits and costs of participating in the subsequent markets and which includes both physical futures contracts and bilateral contracts.
\end{abstract}

Index Terms-spot electricity markets, financial electricity markets, Iberian Electricity Market, stochastic programming, perspective cuts.

\section{INTRODUCTION}

Finding the optimal bid to the day-ahead market (DAM) is a crucial decision in the daily operation of any generation company (GenCo), as DAM is the market where the most important part of the electricity demand is negotiated (78\% in the case of the Iberian Electricity Market (MIBEL)). Moreover, current electricity markets are organized around a variety of markets, both financial (futures contracts (FC), bilateral contracts (BC)) and spot (day-ahead market (DAM), reserve market (RM) and intraday market (IM)) with a strong dependence between them. Therefore, any GenCo aiming to participate in the DAM can no longer find its optimal bid without considering the relation between DAM and the rest of the spot and financial markets. Optimal multimarket electricity bid (OMEB) models are large scale nonlinear combinatorial stochastic optimization models designed to help GenCos to find the optimal offer bid of each one of their generation units in such a complex multimarket framework. Due to the large scale and complexity of the OMEB models, commercial optimization software cannot reach the optimal solution within the time required by the DAM's submission deadline, and more efficient combinatorial nonlinear optimization algorithms are required. The first part of this work presents a new multistage stochastic optimization model for the OMEB problem that find the optimal bid for the DAM taking into account the relation between this spot market and the rest of the market mechanisms (FC, BC, RM, IM) in the Iberian Electricity Market. The proposed methodology provides, for each GenCo's generation unit, the optimal unit commitment, and the optimal bid to the DAM. This optimal bid integrates the negotiated energy of the financial markets (FC and BC), and takes into account the stochastic behavior of the three spot markets (DAM, RM and IM). The second part of the work will present the methodology and computational results of the application of the perspective cuts algorithm to the solution of the OMEB problem. The solution to a real case instance of the OMEB problem corresponding to GenCos operating in the MIBEL will be presented and analyzed.

\section{Literature REVIEW}

The optimal multimarket bidding problem has not been studied as much as the day-ahead bidding problem and there are few research groups that have confronted this problem with stochastic techniques. The work of [1] is one of the first works that defines a bidding strategy for a GenCo participating in a sequence of three short-term markets. The work in [2] considers a multistage stochastic model to decide the unit commitment and the capacity allocation in each market but without any bidding strategy. Furthermore, [3] propose a stochastic model for obtaining the bid curve to be submitted in each market. The most recent contribution, [4], can be consider as an extension of [2], where a risk aversion tool is added together with the satisfaction of the committed bilateral contracts. Contrary to the previous contributions, our approach takes into account the sequence of markets according to the specific characteristics of MIBEL regulation and two different medium-term products, BCs and FCs. The model presented here extends the optimal bid model in [5], [6] and [7] to the multimarket environment. The work in [8] follows the same idea presented in this paper but (a) with a simplified modelization where only linear generation costs were considered and unit commitment was excluded and (c) using commercial 
general purpose optimization software instead of specialized algorithms.

\section{Fundamentals AND HyPOTHESIS}

\section{A. Fundamentals}

The main characteristics of the three electricity markets considered in this work are:

- Day-ahead market: it is the market where the most important part of the energy transactions are negotiated. It takes place the day before the delivery day. It has 24 simultaneous auctions, one for each hour of the next day. The DAM matching process is coordinated with the BCs and the physical FCs as it will be explained later.

- Reserve market: takes place after the DAM matching process. It is an ancillary service market where the participants send bids to increase or decrease the matched energy of the units in the DAM.

- Intraday market: takes place just before and during the delivery day. It is composed of 6 consecutive markets. In these markets the GenCos can either sell or buy electricity, that is, they can participate as buyers or sellers of energy. A specific unit can participate in these markets either if its bids have been matched in the DAM or if it is producing energy to settle BCs.

These three markets are sequentially cleared leading to a multistage stochastic programming problem associated with a set $S$ of scenarios of the DAM, RM and IM market prices $\lambda^{s}=\left\{\lambda_{1}^{D, s} . . \lambda_{24}^{D, s}, \lambda_{1}^{R, s} . . \lambda_{24}^{R, s}, \lambda_{1}^{I, s} . . \lambda_{24}^{I, s}\right\}, s \in S$.

The MIBEL rules force the GenCo to include in the DAM bid process the settlement of the energy from other market mechanisms. In this work, the national bilateral contracts and the futures physical contracts matched at the derivatives market are included. Regarding the physical futures contracts portfolio and the day-ahead bidding mechanism OMEL demands every GenCo to commit the quantity designed to futures contracts through the day-ahead market bidding of the physical units that form each UCP, a pre-stablished subset of the thermal units which will generate the energy to cover the corresponding contract. This commitment is done by the so called instrumental price offer, that is, a sale offer with a bid price of $0 € / \mathrm{MWh}$ (also called price acceptant). Due to the algorithm the market operator uses to clear the day-ahead market, all instrumental price offers will be matched (i.e. accepted) in the clearing process, that is, this energy shall be produced and will be remunerated at the spot price.

Bilateral contracts in the MIBEL has the classical characteristics, they are agreements between a generation company and a qualified consumer to provide a given amount of electrical energy at a stipulated price along a delivering period. The characteristics of the bilateral contracts (energy, price, delivering period) are negotiated before the DAM and the energy that is destined to the $\mathrm{BC}$ is excluded from the DAM bid. Accordingly to the MIBEL rules, the DAM bid of each unit must include the whole available energy not allocated to the $\mathrm{BC}$ contracts.

\section{B. Hypothesis}

The model is built for a price-taker GenCo owning a set of thermal generation units $I$ with startup, shutdown and quadratic generation costs. We will assume some modeling hypothesis about the RM and IM. First, we suppose that all the units in our model are capable of changing their production according to the requirements of the ISO, which means all the available units can participate in the RM. Second, we also suppose that if the GenCo participates in the RM, then it will always bid the automatic generation control (AGC) capacity of the unit, an operational characteristic of each unit that indicates the quantity that the unit is able to increase or decrease in a given time. This hypothesis follows the real behavior of some GenCos observed in the MIBEL. Moreover, we work only with the first IM session, the session in which the greater part of the energy is negotiated. Finally, we suppose that all the energy that is bid to the RM or the IM will be matched. This can be easily forced by some bidding strategies, but this point is not dealt with in this work. These hypothesis do not limit the correct representation of the MIBEL's market sequence and they can be easily changed or adapted to different situations.

\section{MODEL DESCRIPTION}

\section{A. Variables}

For every time period $t \in T$ and thermal unit $i \in I$, the first stage variables of the stochastic programming problem are:

- The unit commitment variables: $u_{t i} \in\{0,1\}, c_{t i}^{u}, c_{t i}^{d}$

- The instrumental price offer bid variables: $q_{t i}$.

- The scheduled energy for futures contract $j$ variables: $f_{t i j}$.

- The scheduled energy for bilateral contract variables: $b_{t i}$. and the second and third stage variables associated with each scenario $s \in S$ are:

- Total generation: $g_{t i}^{s}$

- Matched energy in the day-ahead market: $p_{t i}^{s}$

- Reserve market related variables: $r_{t i}^{s}$

- Intraday market related variable: $m_{t i}^{s}$

\section{B. FCs and BCs Covering Constraints}

Both the physical future and bilateral contracts coverage must be guaranteed:

$$
\begin{array}{lr}
\sum_{i \in I_{j}} f_{t i j}=L_{j}^{F} & \forall j \in F, \forall t \in T \\
\sum_{i \in I} b_{t i}=\sum_{j \in B} L_{j}^{B} & \forall t \in T \\
f_{t i j} \geq 0 & \forall j \in F, \forall i \in I, \forall t \in T \\
0 \leq b_{t i} \leq \bar{P}_{i} u_{t i} & \forall i \in I, \forall t \in T
\end{array}
$$

\section{Reserve Market Constraints}

By hypothesis the model for the RM assumes that if the unit bids to the RM, it will bid its fixed AGC capacity, $\varrho_{i}(\mathrm{MW})$. Therefore the only decision to be optimized is whether the unit participates in the RM or not. It is known that a unit can only use its AGC capacity if its generation level is constant; in other 
words, the unit is not increasing or decreasing its production in the corresponding interval or, equivalently, that the production level $g_{t i}^{s}$ has not changed between two consecutive intervals. For all intents and purposes, the GenCo delegates its ramping capacity to the ISO. The binary variable $r_{t i}^{s}$ is introduced to trace this situation, being that $r_{t i}^{s}=1$ whenever $g_{t i}^{s}=g_{(t-1), i}^{s}$ and $r_{t i}^{s}=0$ otherwise.

$$
\begin{array}{ll}
g_{t i}^{s}-g_{(t-1), i}^{s} \leq\left(1-r_{t i}^{s}\right) \bar{P}_{i} \quad \forall i \in I, \quad \forall t \in T, \forall s \in S \\
g_{t i}^{s}-g_{(t-1), i}^{s} \geq\left(1-r_{t i}^{s}\right)\left(-\bar{P}_{i}\right) \quad \forall i \in I, \quad \forall t \in T, \forall s \in S
\end{array}
$$

Moreover, uncommitted units $\left(u_{t i}=0\right)$ cannot bid to the RM:

$$
r_{t i}^{s} \leq u_{t i} \quad \forall t \in T, \forall i \in I, \forall s \in S
$$

\section{Matched Energy Constraints}

The MIBEL's rules affecting the day-ahead market establishes a given relation between the variables representing the energy of the bilateral contracts $b_{t i}$, the energy of the future contracts $f_{t i j}$, the instrumental price offer bid $q_{t i}$ and the matched energy $p_{t i}^{s}$. This relation can be formulated by means of the following set of constraints:

$$
\begin{array}{lr}
p_{t i}^{s} \leq \bar{P}_{i} u_{t i}-b_{t i} & \forall i \in I, \quad \forall t \in T, \forall s \in S \\
p_{t i}^{s} \geq q_{t i} & \forall i \in U_{t}, \quad \forall t \in T, \forall s \in S \\
q_{t i} \geq \underline{P}_{i} u_{t i}-b_{t i} & \forall i \in I, \forall t \in T \\
q_{t i} \geq \sum_{j \mid i \in I_{j}} f_{t i j} & \forall i \in I, \forall t \in T
\end{array}
$$

where:

- (8) and (9) ensures that if a unit is on, the matched energy $p_{t i}^{s}$ will be between the instrumental price bid $q_{t i}$ and the total available energy not allocated to a BC.

- (10) and (11) guarantee respectively that the minimum generation output of the committed units will be matched, and that the contribution of the unit to the FC coverage will be included in the instrumental price bid.

\section{E. Total Generation and Intraday Market Constraints}

Finally, the total generation level of a given unit $i, g_{t i}^{s}$, is defined as the addition of the allocated energy to the BC, plus the matched energy in the DAM and IM $\left(p_{t i}^{s}\right.$ and $m_{t i}^{s}$ respectively).

$$
g_{t i}^{s}=b_{t i}+p_{t i}^{s}+m_{t i}^{s} \quad \forall t \in T, \forall i \in I, \forall s \in S
$$

The model considers the possibility of either selling $\left(m_{t i}^{s}>0\right)$ or buying $\left(m_{t i}^{s}<0\right)$ energy to the IM. The total generation $g_{t i}^{s}$ must remain within the operational limits $\underline{P}_{i}$ and $\bar{P}_{i}$. But if we participate in the RM, the total generation limits change because of the energy that we must reserve in order to be able to produce it at the moment that the ISO requests:

$$
\underline{P}_{i} u_{t i}+\varrho_{i} r_{i t}^{s} \leq g_{t i}^{s} \leq \bar{P}_{i} u_{t i}-\varrho_{i} r_{i t}^{s} \quad \forall t \in T, \forall i \in I, \forall s \in S
$$

\section{F. Nonanticipativity Constraints}

Nonanticipativity constraints impose that the value of the second and third stage variables will be the same for those stages and scenarios sharing the same value of the random variables $\lambda^{s}$ :

$$
\begin{aligned}
& g_{t i}^{s}=g_{t i}^{\hat{s}} \quad \forall s, \hat{s}:\left(\lambda^{D, s}=\lambda^{D, \hat{s}}\right), \forall t \in T \\
& r_{t i}^{s}=r_{t i}^{\hat{s}} \quad \forall s, \hat{s}:\left(\left(\lambda^{D, s}, \lambda^{R, s}\right)=\left(\lambda^{D, \hat{s}}, \lambda^{R, \hat{s}}\right)\right), \forall t \in T
\end{aligned}
$$

where (14) models the nonanticipativity constraints for the DAM stage and (15) models the nonanticipativity constraints for the RM stage.

\section{G. Unit commitment}

Following [9], let $u_{i}^{t}$ be the first-stage binary variable expressing the off-on operating status of the $i^{t h}$ unit and $c_{t i}^{u}, \quad c_{t i}^{d}$, continuous variables representing the startup and shutdown cost, respectively, of unit $i$ in interval $t$. Additionally, constant $G_{i}$, will be the number of periods that unit $i$ must be initially online, due to its minimum up-time $t_{i}^{\text {on }}$, and $H_{i}$, will be the number of periods that unit $i$ must be initially offline, due to its minimum down-time $t_{i}^{\text {down }}$. The following set of constraints conveniently models the start-up and shutdown costs and the minimum operation and idle time for each unit (see [9] for details):

$$
\begin{aligned}
& c_{t i}^{u} \geq c_{i}^{o n}\left[u_{t i}-u_{(t-1), i}\right] \quad \forall t \in T \backslash\{1\}, \forall i \in I \\
& c_{t i}^{d} \geq c_{i}^{o f f}\left[u_{(t-1), i}-u_{t i}\right] \quad \forall t \in T \backslash\{1\}, \forall i \in I \\
& \sum_{j=n}^{G_{i}}\left(1-u_{j i}\right)=0 \\
& \forall i \in I \\
& \sum_{j=1}^{H_{i}} u_{j i}=0 \\
& \forall i \in I \\
& \sum_{n=t}^{t+t_{i}^{o n}-1} u_{n i} \geq t_{i}^{o n}\left[u_{t i}-u_{(t-1), i}\right] \\
& \forall t=G_{i}+1, \ldots,|T|-t_{i}^{o n}+1 \forall i \in I \\
& t+t_{i}^{\text {off }}-1 \\
& \sum_{n=t}\left(1-u_{n i}\right) \geq t_{i}^{o f f}\left[u_{(t-1), i}-u_{t i}\right] \\
& \forall t=H_{i}+1, \ldots,|T|-t_{i}^{o f f}+1 \forall i \in I \\
& \sum_{n=t}^{|T|}\left(u_{n i}-\left[u_{t i}-u_{(t-1), i}\right]\right) \geq 0 \\
& \forall t=|T|-t_{i}^{o n}+2, \ldots,|T| \forall i \in I \\
& \sum_{n=t}^{|T|}\left(1-u_{n i}-\left[u_{(t-1), i}-u_{t i}\right]\right) \geq 0 \\
& \forall t=|T|-t_{i}^{o f f}+2, \ldots,|T| \forall i \in I
\end{aligned}
$$




\section{H. Objective Function}

The quadratic function that represents the expected benefits of the GenCo:

$$
\begin{aligned}
E_{\lambda} & {\left[B\left(g, p, m, r, u, c^{u}, c^{d}\right)\right]=} \\
= & \sum_{t \in T}\left[\sum_{j \in F}\left(\lambda_{j}^{F}-\bar{\lambda}_{i}^{D}\right) L_{j}^{F}+\sum_{j \in B} \lambda_{j}^{B} L_{j}^{B}\right] \\
- & \sum_{t \in T} \sum_{i \in I}\left[c_{t i}^{u}+c_{t i}^{d}+c_{i}^{b} u_{t i}\right] \\
+ & \sum_{t \in T} \sum_{i \in I} \sum_{s \in S} P^{s}\left[\lambda_{t}^{D, s} p_{t i}^{s}+\lambda_{t}^{R, s} \varrho_{i} r_{t i}^{s}+\lambda_{t}^{I, s} m_{t i}^{s}\right. \\
& \left.\quad-\left(c_{i}^{l} g_{t i}^{s}+c_{i}^{q}\left(g_{i t}^{s}\right)^{2}\right)\right]
\end{aligned}
$$

where:

(24) corresponds to the incomes of the FCs and the BCs and is a constant term.

(25) is the on/off fixed cost of the unit commitment of the thermal units, deterministic and independent of the realization of the random variable $\lambda_{t}^{D}=\left\{\lambda_{t}^{D}, \lambda_{t}^{R}, \lambda_{t}^{I}\right\}$.

(26) represents the expected value of the benefits from the DAM, the RM and the IM. The first term, $\lambda_{t}^{D, s} p_{t i}^{s}$, computes the incomes from the DAM based on a value $p_{t i}^{s}$ of the matched energy. The second term, $\lambda_{t}^{R, s} \varrho_{i} r_{t i}^{s}$ computes the incomes from bidding the AGC capacity to the RM. The third term, $\lambda_{t}^{I, s} m_{t i}^{s}$ computes the incomes or costs from the IM, depending on the sign of $m_{t i}^{s}$. Finally, the term between brackets corresponds to the expression of the quadratic generation costs with respect to the total generation of the unit, $g_{t i}^{s}$.

Then, the objective function $f(x)$ to be minimized in our model is:

$$
\begin{gathered}
f\left(g, p, r, m, u, c^{u}, c^{d}\right)=\sum_{i \in I} \sum_{t \in T}\left(c_{t i}^{u}+c_{t i}^{d}+c_{i}^{b} u_{t i}\right. \\
+\sum_{s \in S} P^{s}\left[c_{i}^{l} g_{t i}^{s}+c_{i}^{q}\left(g_{t i}^{s}\right)^{2}-\left(\lambda_{t}^{D, s} p_{t i}^{s}\right)\right. \\
\left.\left.-\left(\lambda_{t}^{R, s} r_{t i}^{s} \varrho_{i}\right)-\left(\lambda_{t}^{I, s} m_{t i}^{s}\right)\right]\right)
\end{gathered}
$$

where $\lambda_{t}^{D, s}, \lambda_{t}^{R, s}, \lambda_{t}^{I, s}$ are the price scenarios for the $t^{t h}$ dayahead, reserve or intraday market respectively.

\section{Optimal multimarket electricity bid model (OMEB)}

The final Optimal electricity multimarket bid (OMEB) model developed in the previous sections is:

$$
(\text { OMEB })\left\{\begin{array}{lll}
\min & f\left(g, p, r, m, u, c^{u}, c^{d}\right) & \\
\text { s.t. } & & \\
& \text { Eq. }(1)-(4) & \text { BC and FC } \\
& \text { Eq. }(5)-(6) & \text { RM } \\
& \text { Eq. }(8)-(11) & \text { DAM } \\
& \text { Eq. }(12)-(7) & \text { Tot. gen. } \\
& \text { Eq. }(16)-(23) & \text { UC }
\end{array}\right.
$$

This program corresponds to a mixed linearly constrained minimization problem with a convex quadratic objective function with a well-defined global optimal solution.

\section{Perspective Cuts}

In order to solve OMEB model by commercial MILP software, the quadratic part of the objective function must be linearized. Since the sum of the probabilities $P^{s}$ equals one, we can include the products $c_{t i}^{b} u_{t i}$ in the quadratic parenthesis for each block $(i, t, s)$ in this way:

$$
c_{i}^{q}\left(g_{t i}^{s}\right)^{2}+c_{i}^{l} g_{t i}^{s}+c_{i t}^{b} u_{t i},
$$

where the variables $u_{t i}$ are binary. For notational simplicity the indices will be omitted in the rest of this section. The issue is then how to best represent the quadratic function

$$
f(g, u)=c^{q} g^{2}+c^{l} g+c^{b} u
$$

by means of a piecewise-linear one. There is an effective way based on ideas developed by [10]. Note that, as $u$ is binary and $u \underline{P} \leq g \leq u \bar{P}$, we have

$$
f(g, u)= \begin{cases}0, & \text { if } u=0 \\ f(g)=c^{q} g^{2}+c^{l} g+c^{b}, & \text { if } u=1\end{cases}
$$

Moreover, when we use the branch and cut methods in order to find lower bounds for the optimal value, we solve continuous relaxations of the mixed integer linear problem, i.e. with $u \in$ $[0,1]$. Therefore, a natural question is whether we can obtain a convex function with an tighter epigraph for $f(g, u)$, which can be used to calculate those lower bounds, this leads us to take into account the convex envelope of $f(g, u)$. As it is shown in [10] the convex envelope is

$$
h(g, u)=\left\{\begin{array}{ll}
0, & \text { if }(g, u)=(0,0) \\
\frac{c^{q} g^{2}}{u}+c^{l} g+c^{b} u, & \left\{\begin{array}{c}
\text { if } u \underline{P} \leq g \leq u \bar{P}, \\
\text { for } u \in(0,1]
\end{array}\right. \\
+\infty, & \text { otherwise }
\end{array}\right\}
$$

This function is the perspective-function $\breve{f}(g, u)=u f(g / u)$ of $f(g)$, with $u$ limited to be in $[0,1]$, which is known to be convex if $f(g)$ is convex, see [11]. In addition, to show that $h$ is a tighter objective function than $f$ for the continuous relaxation it is enough to compare (29) and (30) for $0<u \leq 1$. Also, for $g \in[\underline{P}, \bar{P}]$ and $u \in[0,1]$ it can be showed that the maximum value of $h(g, u)-f(g, u)$ over the domain of both functions (defined by the pyramid having as base $[\underline{P}, \bar{P}] \times\{1\}$ and vertex $[0,0])$ is $c^{q} \bar{P}^{2} / 4$, attained at $(\bar{P} / 2,1 / 2)$; i.e. $h$ penalizes the highest non-integrality in the domain. Nevertheless, due to the strong nonlinearity and the nondifferentiability of $h(g, u)$ at $(0,0)$, it is not practical to use it as the objective function instead of $f(g, u)$. A way of overcoming this difficulty is to replace $h(g, u)$ with the pointwise supremum of affine functions, which is possible because the convexity of $h$. As is showed in [10], by means of the subgradient inequality

$$
h(g, u) \geq h(\widehat{g}, \widehat{u})+\left(s_{1}, s_{2}\right)((g, u)-(\widehat{g}, \widehat{u})),
$$

where $\left(s_{1}, s_{2}\right) \in \partial h(\widehat{g}, \widehat{u})$, the epigraph of $h$ is defined by the subset of

$$
\{(v, g, u) \mid u \underline{P} \leq g \leq u \bar{P} \text { and } 0 \leq u \leq 1\}
$$


that is solution of this infinite linear-inequality system

$$
v \geq\left(2 c^{q} \widehat{g}+c^{l}\right) g+\left(c^{b}-c^{q} \widehat{g}^{2}\right) u, \quad \text { taking } \quad \widehat{g} \in[\underline{P}, \bar{P}] .
$$

For each $\widehat{g}$ we have an inequality so-called a perspective cut (PC), which is the unique supporting hyperplane to the function passing by $(0,0)$ and $(\widehat{g}, 1)$. Consequently, $P C$ formulation (PCF) consists of using these perspective cuts to construct an objective function that is the point-wise maximum of the linear functions of these hyperplanes, i.e. it is a polyhedral function. A small set of initial PCs is chosen to solve the problem with the continuous relaxation. Then, given a solution $\left(v^{*}, g^{*}, u^{*}\right)$ corresponding to an approximation of $h$ by a finite number of perspective cuts, when $u^{*}>0$, it is tested to know if this solution fulfills (31) with $\widehat{g}=g^{*} / u^{*}$; otherwise, this supporting hyperplane is a new perspective cut to be added to PCF. Thus, additional cuts are then dynamically generated when necessary.

\section{NUMERICAL EXAMPLES}

The OMEB model (28) has been implemented and solved with CPLEX 12.0 [12] using the ad-hoc implementation of the perspective cuts algorithm described in Section IV. It has been solved using a SunFire X2200 with 32 Gb of RAM memory and two dual core processors AMD Opteron 2222 at $3 \mathrm{GHz}$. The total execution times for the perspective cuts methodology are shown in Table I. It must be stressed that all these problems were unsolvable with CPLEX (the execution aborted with an internal error after several days of execution).

\section{A. Scenario generation and reduction}

All the available historical data of the sequence of market prices has been reduced in order to obtain suitable scenario sets. Initially, all the instances are equiprobable and, after applying the reduction algorithm [13], the different subsets of scenarios and the respective probabilities are obtained. The main computational characteristics for each reduced set of scenarios are in Table I. It can be observed that both the objective function and the variation of the optimal value of the variables (measured through the index $\frac{\left\|x^{s}-x^{180}\right\|}{\left\|x^{180}\right\|}$ ) stabilizes after 50 scenarios. Considering the computational burden introduced by the increase of second and third stage binary variables as the number of scenarios grows, we conclude that 50 scenarios retain enough information to obtain suitable results.

\section{B. Case Study}

A set of computational tests has been performed in order to validate the proposed model. The instances used in the test have 9 thermal units. Its technical values can be obtained from the authors upon request. One of the objectives of the tests is to study the influence of the sequence of markets in the DAM bid. As it has been explained, the DAM bid of the GenCo will be fixed by the quantity committed to bilateral contracts, that will be excluded from the DAM bid, and the quantity committed to futures contracts, which must be bid at the instrumental price (see [6]). Thus, we focus on the two variables that represent these quantities in order to study its optimal value when taking
TABLE I

OPTIMIZATION CHARACTERISTICS OF THE CASES STUDIES AND RESULTS FOR DIFFERENT NUMBER OF SCENARIOS.

\begin{tabular}{cccccc}
\hline$|S|$ & c.v. & b.v. & CPU(s) & Objective function & $\frac{\left\|x^{s}-x^{180}\right\|}{\left\|x^{180}\right\|}$ \\
\hline 25 & 19680 & 6240 & 612 & 89230500 & 1,000 \\
50 & 37680 & 12240 & 3093 & 88268300 & 0,001 \\
75 & 55680 & 18240 & 12316 & 88624200 & 0,002 \\
100 & 73680 & 24240 & 25728 & 88177400 & 0,001 \\
120 & 88080 & 29040 & 32570 & 88209200 & 0,001 \\
140 & 102480 & 33840 & 60030 & 88318100 & 0,002 \\
160 & 116880 & 38640 & 74865 & 88298800 & 0,002 \\
180 & 131280 & 43440 & 93532 & 88209200 & \\
\hline \multicolumn{5}{c}{$|T|=24 ;|I|=9$}
\end{tabular}

into account, or not, the sequence of markets. The optimal value of these two variables can be observed in Figure 1. This figure represents the economic dispatch of the bilateral contracts, i.e., the quantity each unit commits to the bilateral contracts for each interval $t$, and the quantity to cover the futures contracts. It can be also observed the big differences among the optimal economic dispatch if we include the RM and the IM in the optimization model (Figure 1(a)) or not (Figure 1(b)). On the one hand, if a unit participates into the RM market, it must reserve a part of its participation and thus cannot use it to cover the medium-term products (see, for instance, Unit 2 at intervals 3, 7 or 8). On the other hand, they could buy or sell energy into the IM, and this can change the settlement of the medium-term products. Those differences will lead to different offer curves for each unit and interval.

Finally, although second and third stages variables are not related with any actual decision, as they differ from scenario to scenario, it could be interesting to observe the behavior of the GenCo in the IM, where the GenCo can submit either sell or purchase bids, depending on the sign of variable $m_{t i}^{s}\left(m_{t i}^{s}>0\right.$ and $m_{t i}^{s}<0$ respectively). Figure 2 represents the bidding energy for a given unit throughout the 24 hourly auctions of the IM market day at two different scenarios. It can be observed that, depending on the auction, the GenCo either buys or sells energy, or it does not participate in the IM.

\section{CONCLUSiON}

This work has developed a new quadratic mixed-integer stochastic programming model, to assist to the optimization of the day-ahead bid with futures and bilateral contracts taking into account the reserve and the intraday market. The optimal solution of our model determines the optimal instrumental price bidding strategy and the optimal economic dispatch for the BCs and the committed FCs for each hour. The model maximizes the expected benefits of the sequence of electricity markets while satisfying the thermal operational constraints and the MIBELs rules. The results of the computational tests validate the model and show the influence of market sequence on the optimal bidding strategy of the GenCo, as well as the short-sight effect of optimizing the DAM bid without taking into account the possibilities of the next markets. 
(a)
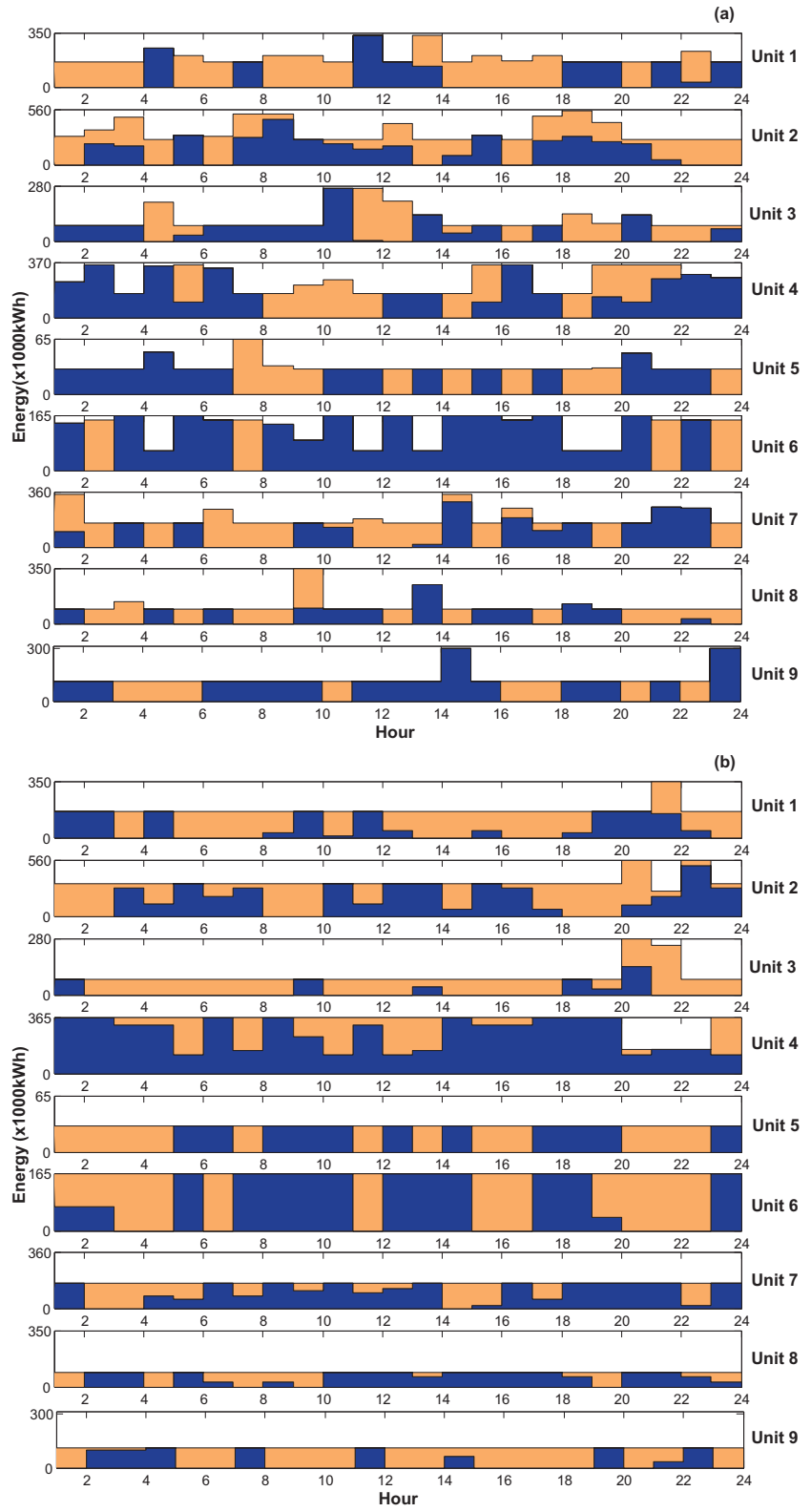

Fig. 1. Economic dispatch of bilateral and futures contract, $b_{t i}$ (blue) and $q_{t i}$ (orange) . (a) Taking into account market sequence (b) With the DAM only.

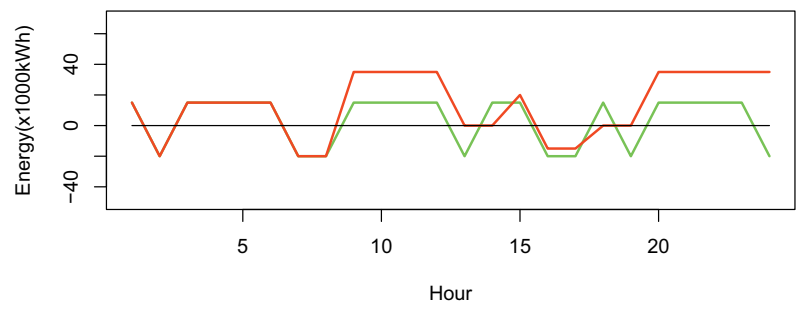

Fig. 2. Energy send to the IM by Unit 1 in two different scenarios.

\section{REFERENCES}

[1] M. A. Plazas, A. J. Conejo, and F. J. Prieto, "Multimarket optimal bidding for a power producer," IEEE Transactions on Power Systems, vol. 20, no. 4, pp. 2041-2050, 2005.

[2] C. Triki, P. Beraldib, and G. Grossc, "Optimal capacity allocation in multi-auction electricity markets under uncertainty," Computers \& Operations Research, vol. 32, no. 2, pp. 201-217, 2005.

[3] A. Ugedo, E. Lobato, A. Franco, L. Rouco, J. Fernandez-Caro, and J. Chofre, "Strategic bidding in sequential electricity markets," in IEEE Proceedings Generation, Transmission and Distribution, vol. 153, no. 4 2006, pp. 431-442.

[4] R. Musmanno, N. Scordino, C. Triki, and A. Violi, "A multistage formulation for generation companies in a multi-auction electricity market," IMA Journal of Management Mathematics, 2009.

[5] C. Corchero and F.-J. Heredia, "A stochastic programming model for the thermal optimal day-ahead bid problem with physical futures contracts," Computers \& Operations Research (in press), 2011.

[6] F. J. Heredia, M. J. Rider, and C. Corchero, "Optimal bidding strategies for thermal and generic programming units in the day-ahead electricity market," IEEE Transactions on Power Systems, vol. 25, no. 3, pp. 1504 $-1518,2010$

[7] _ - "A stochastic programming model for the optimal electricity market bid problem with bilateral contracts for thermal and combined cycle units," Annals of Operations Research (in press), 2011.

[8] C. Corchero and F. J. Heredia, "Optimal day-ahead bidding in the mibel's multimarket energy production system," IEEE Proceedings of the $7^{\text {th }}$ Conference on European Energy Market, vol. 1, pp. 1-6, 2010.

[9] M. Carrin and J. M. Arroyo, "A computationally efficient mixed-integer linear formulation for the thermal unit commitment problem," IEEE Transactions on Power Systems, vol. 21, no. 3, pp. 1371-1378, 2006.

[10] A. Frangioni and C. Gentile, "Perspective cuts for a class of convex 0-1 mixed integer programs," Mathematical Programming, vol. 106, pp. 225-236, 2006.

[11] J. B. Hiriart-Urruty and C. Lemarchal, Convex Analysis and Minimization Algorithms IIAdvanced Theory and Bundle Methods, ser. A Series of Comprehensive Studies in Mathematics. Springer- Verlag, 1993, vol. 306.

[12] CPLEX, "Cplex optimization subroutine library guide and reference. version 12.0," 2008, cPLEX Division, ILOG Inc., Incline Village, NV, USA.

[13] N. Grwe-Kuska, H. Heitsch, and W. Rmisch, "Scenario reduction and scenario tree construction for power management problems," in Power Tech Conference Proceedings, 2003 IEEE Bologna, vol. 3, 23-26 June 2003. 